Zä̈ngerle M. Grundriss der Botanik für den Unterricht an mittleren und höheron Lehranstalten. 2. Aufl. München (G. Taubald). 8". 174 S. Ill. - $2 \cdot 60 \mathrm{Mk}$.

\title{
Dr. Günther Ritter Beck v. Mannagetta: Flora von Niederösterreich.
}

\section{Theil.}

\section{Besprochen von Dr. R. v. Wettstein (Wien).}

(sch]ust. $\left.{ }^{1}\right)$

Ferner möge die vom Verfisser vorgenommene Unterscheidung engerer Formenkreise innerhalb der Art kurz besprochen werden. Immer mehl bricht sich die Ueberzeugung Bahn, dass die Unterscheidung "kleiner" Formen für die Systematik von grosser Wichtigkeit ist, da diese "kleinen" Formen in der Regel auch die relativ jüngsten, noch am klarsten die Verhältnisse der Entwickelung zeig'en und daher von dieser Basis aus die Entwickelungsgeschichte der Pflanzenwelt inductiv studirt werden kann. Referent kann daher dem Verfasser nur beipflichten, wenn er die in seinem Florengebiete zu beobachtenden Formen constatirt. Dagegen möchto er sich gegen die Art and Weise, in welcher diese Formen vielfach angeführt werden, aussprechen. Man kann diesbezüglich ron dem Bearbeiter einer Landesflora nicht gerade specielle Untersuchungen jeder einzelnen Form verlangen, man kann aber beanspruchen, dass 1. in Fällen, in denen durch gründliche vorliegende Arbeiten Klarheit geschaffen wurde, nicht durch kritiklose Nebeneinanderstellung wieder völlige Unklarheit gebracht wird, dass 2. nicht $\mathrm{mehr}$ von einer Pflanze behauptet wird, als sich beweisen lässt.

In diesen beiden Beziehungen findet sich Manches in dem Buche, mit dem sich der Referent nicht einverstanden erklären kann. Nur an einem Beispiele möge dies erläutert werden.

Ad 1. Verfasser zählt auf Seite 78 folgende "Abänderungen" von Seslevia coerulea Ard. auf: $\alpha$. calcarea Opiz, $\beta$. Heufteriana Schur,

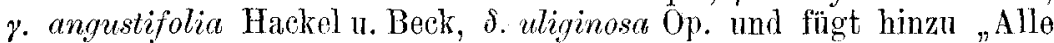
A bänderungen in einander übergehend". Darnach muss Jedermann den Findruck erhalten, als seien die vier aufgezählten Formen gelegentlich auftretende, durch äussere Einflüsse oder irgend eine andere Ursache hervorgerufene, systematisch gleichwerthige Variationen ohne Constanz. Dem ist nun durchaus nicht so. Ich glaube in meiner kleinen Arbeit über "Sesleria coerulea L." (Verh. d. k. k. zool.-botan. Gesellsch. 1888) nachgewiesen zu haben, dass unter dem Namen "Sesleria coerulea" z w $\theta$ i wesentlich verschiedene, ganz constant verschieden bleibende. Pflanzen zusammengefasst werden, die sich bei allem Variationsvermögen des Individuums stets unterscheiden lassen und dass eine dieser beiden Pflanzen die von Linné als "coerulea" beschriebene ist.

I) Vergl. diese Zeitschr. 1891, Nr. 1. 
Von diesen beiden Pflanzen gilt der Ausspruch des Verfassers "gehen ineinander über", wenigstens in dem von ihm angewendeten Sinne $e^{1}$ gewiss nicht. Ich habe vor und nach der Veröffentlichung meiner Abhandlung die Pflanzen an zahlreichen Standorten studirt; ich habe ausreichende, Jedermann zugängliche Culturversuche im botanischen Garten der Wiener Universität durchgeführt und kann auf das Bestimmteste behaupten, dass die beiden Pfanzen sich niemals ineinander überführen lassen.

Des Verfassers Behauptung, dass dies zwei ineinander übergehende gelegentliche Abänderungen einer Pflanze seien, lässt sich nur dadurch erklären, dass er sie, trotz meiner deutlichen Beschreibung, nicht genügend kennt. Und dafür, dass diese Erklärung richtig ist, spricht der Umstand, dass er die eine derselben, nämlich die Linnésche Sesleria coerulea $(==$ uliginosa Op.) nicht erkannte, als er sie bei Moosbrunn sammelte, sondern sie für S. ITeufleriana Schur hielt. In Folge dessen findet sich auch in dem vorliegenden Buche diese neben S. uliginosa anfgeführt. — Ich kenne die Flora der Moosbrunner Sumpfwiesen sehr gut, habe speciell dort die Seslerien studirt und kann auf das Bestimmteste behaupten, dass dort nur Sesleria coerulea (Linné) Wettst. (= uliginosa Op.) vorkommt. Ich kenne auch S. Henfleriana Schur aus Original-Exomplaren sehr gut, weiss, wie sie sich zu S. coerulea (L.) verhält and kann ebenso bestimmt behaupten, dass sie bei Moosbrunn fehlt. S. Heufleriana Beck ist daher S. coerulea (L.) oder eine Standortsform derselben.

Die vierte vom Verfasser angeführte Form: $\gamma$. angustifolia Hackel u. Beck dürfte, den Verbältnissen an den Standorten nach zu urtheilen, wirklich in S. varia (Jacq.) Wettst. (= S. calcarea Op.) übergehen, niemals aber in Sesleria coerulea $\mathrm{I}_{\text {. }}$; sie gehört mithin als Varietät zur erstgenannten.

Es ergibt sich demnach, dass die nioderösterreichischen Seslerien sich folgendermassen verhalten. Es gibt $z$ wei systematisch gleichwerthige Pflanzen, die S. coerulea (L.) und die S. varia (Jacq.), welche entweder als zwei coordinite Arten oder als gleichwerthige Unterformen einer Art angeführt werden müssen. ${ }^{2}$ ) Zujederderselben gehörtals Form von geringerem systematischon Werthe (Standortsvarietät?) je eine Pflanze, zu der ersteren eventuell die vom Verfasser als $S$. Heuferiana bezeichnete, zur zweiten S. angustifolia Hackel u. Beck., ${ }^{3}$ )

Diese vier Pflanzen aber als gleichwerthige Abänderungen aufzuführen, wie es Verfasser that, ist unrichtig und darnach angethan, die schon sichergestellten Kenntinisse wieder zu verwirren,

1) Nämlich wirkliche Uebergänge und nicht Hybride.

?) Ersteres hätte Verfasser thun müssen, wenn ihn dieselben Anschauungen, die ihn bei anderen Familien leiteten (z. B. Pinus aliginowa Neum. und $P$. pseudopumilio Willk., Betul, polundate Celak. ind B. Carpaticu W. K. als eigene Arten!), bei den Gramineen inassgebenl gewesen wäroll.

$\left.{ }^{3}\right)$ Solcher Formen gibt es abar noch mehr; vergl. diesbezicglich z. B. Schur in Verh. d. zool.botan. Gusellsch. 1830. 
zudem wird ron ihnen durch den Zusatz "alle Abänderungen gehen ineinander über" etwas behauptet, was nicht bewiesen wurde.

Reforent hätte diese tadelnde Bemerkung, die in gleicher Weise für manche andere Art gilt, nicht gemacht, wenn Verfasser den schon erwähnten, bei einer rein floristischen Arbeit durchaus nicht abweisbaren Standpunkt eingenommen hätte, kleinere Formen kritiklos grösseren anzureihen. Sie wurden aber nöthig, nachdem der Verfasser durch seine Bemerkungen und durch sein Verhalten in anderen l'ällen bewies, dass er Kritik anwenden wollte, so dass seinen Anordnungen ein grösseres Gewicht beigelegt werden konnte.

Die Unterscheidung und Benennung mancher minutiösen, geradezu Gandoger'schen Form, wio z. B. der Formen rubra, alba, bracteata bei Silla bifolia L., der Formen rosea und viridans bei Anemone nemorosa L. etc. wäre im Interesse der Vermeidung überflüssiger Nomenclatur und auch aus dem Grunde entbehrlich gewesen, weil dadurch der systematische Werth anderer, in gleicher Weise angeführter "Abänderungen" gewaltig herabgedrückt wird.

Ad 2. Verfasser hat im Anschlusse an Hackel u. a. jene Nomenclatur angewendet, dass für eine der Unterformen der Speciesnamen bleibt und diese als die typische bezeichnet wird. Er hat daher bei vielen Arten eine Form "typica" genannt. Verfasser hat zunächst dieses Princip in seiner "Monographie der Gattung Orobanche" angewendet und nun auf die vorliegende Flora übertragen. Er hat dabei übersehen, dass "si duo faciunt idem, non est idem". Der Monograph hat, wenn seine Arbeit nicht eine blosse Compilation sein soll, die Pflicht, sich über den systematisehen Werth der von ihm unterschiedenen Formen klar zu werden; er kann eine der Formen als typische bezeichnen, wenn er zur Einsicht kommt, dass sio jener am nächsten kommt, die als Ausgangspunkt der anderen anzusehen ist. - Ganz anders der Florist. Wenn ein Florist ohne die Gesammtheit der Formen in Betracht zu ziehen, eine Form herangreift und diese, nur um das Nomenclaturprincip zur Durchführung zu bringen, als typische bezeichnet, so begeht er damit den schon oben erwähnten Fehler, indem er von einer Pflanze mehr als sicher hinstellt, als er beweisen kann.

Wenn es also schon nicht möglich ist, den Ausdruck "typica" in dem angedeuteten Sinne zu gebraucben, so hätte seine Anwendung, wenn auch nicht wissenschaftlich, so doch in Hinblick auf die Nomenclatur, immerhin eine Berechtigung, ${ }^{1}$ ) wenn damit die rom Autor der "Art" gemeinte Form bezeichnet wird, um ihr auf alle Fälle ihren ursprünglichen Namen zu erhalten. Aber auch dies thut Verfasser nicht, denn in diesem Falle hätte er beispielsweise bei Sesleria coerulea Ard. seine Form calcarea Op. als "typica"

"Trotzdem halte ich die Anwendung des Wortes "typisch" in doppeltem Sinne, einmal zur Bezeichnung der muthmasslich usprünglichen Form, ein andermal zur Bezeichnnng jener Form, die der Autor der Art meinte, für unzulässig. 
bezeichnen müssen, er hätte bei Saponaria officinalis L. die Form $\beta$. glaberrima Ser. und nicht seine Form $\alpha$, typisch" nennen. müssen u. s. w. Wir sehen also, dass der Verfasser bald die eine, bald die andere Form herausgriff und als die "typische" hinstellte, damit aber in den bezeichneten Fehler verfiel.

In Kürze möchte jch noch die vom Verfasser angewendete Un torschoidung und Benenung der Hybriden besprechen. Man kann in dieser Hinsicht, wio bekannt, einen verschiedenen Standpunkt eillnehmen, für jeden derselben nicht unwichtige Gründe anführen; nur ein Vorgehen, wie es Verfasser einschlägt, ist nicht zu rechtfertigen. Er geht nämlich von dem Grundsatze aus, dass eine Hybride als neu zu beschreiben und zu benennen ist, wenn sie den bisher existirenden Beschreibungen (auch für Hybride derselben Combination nicht entspricht. Es ist dieser Standpunkt grundverschieden von jenem, welcher auf der Beobachtung fusst, dass zwischen zwei Pflanzen zwei Hybride möglich sind (eine der einen, eine zweite der anderen Stammart näher stehende). Der Vorfasser hält nur die vorliegenden Beschreibungen für massgebend und erk]ärt es dahor als zulässig, dass zwischen 2 Stammarten mohrere, ja unbegrenzt viele Hybride beschrieben und benannt werden kïmen, soforne uur ihre Charakteristik nicht den Beschreibungen schon beobachteter Hybriden entsprechend ist. In Folge dieses Standpunktes finden wir beispielsweise zwischen Salix viminalis und S. purpurea vier Hybride beschrieben und zahlreiche im Gebiete beobachtete Formen mit neuen Namen versehen.

Dem gegenüber sei Folgendes bemerkt: Jeder Botaniker weiss, dass die Individuen einor Pflanzenart verschieden sind, daher nicht alle Individuen einer anf Grund eines Individumms angefertigten Beschreibung vollkommen entsprechen können; es ist loicht einzusehen, dass dies bei Hybriden im erhöhten Masso der Fall sein muss, welche ja die Varjabilität zweier Arton voreinigon. Man kann daher wissensehaftlich nur den ganzen Formenkreis zwischen zwei Arten mit einem Namen belegen, höchstens dann, wenn eine gewisse Constanz im Auftreten sich wahmehmen lüsst, die der einen Stammform näher stehende Pflanze von der dor anderen mehr ähnelnden unterschejden. Wenn man jedoch unbekümmert darum, jedes Exemplar einer Hybriden, das einer auf Grund ejnes anderen Exemplares derselben Combination angelegten Beschreibung vieht entspricht, neu benennt und besehreibt, dann benennt and beschreibt man Individuen; und auf diesem Standpunkt ist der Verfasser angelangt.

Referent will damit die Besprechung des Buches beschliessen. Mit Absicht wurden in den vorstehenden Zeilen Einzelheiten nur dann berïhrt, wenn deren Besprechung zur Bestätigung einer ausgesprochenen Ansicht nöthig war; wenn Referent sich in dieser Hinsicht eine Beschränkung auferlegte, so geschah dies nicht aus Mangel solcher zur Besprechung geeigneter Einzelheiten, sondern insbesondere aus zwei Gründen. Einerseits waren es nicht einzelne 
Angaben, dio ilın zu der vorstehenden Kritik zwangen; Irrthüner im Einzelnon onthalten auch manche der besten Bücher; es wäre engherzig in Aubetracht ejner grösseren Arbeit sich allzuseln all solche zu halten. Andererseits werden die floristisch-systematischen Arbeiten der nächsten Zeit ohnedies Gelegenheit bieten auf solche Einzelheiten ${ }^{1}$ ) einzugehen. Was den Referenten zu den vorstehenden Zeilen zwang, das. Waren die Pijucipien und Anschaungen, welche bei Abfassung des Buches massgebend waren, das war die Pflicht des Referonten vor einer Richtung der Floristik zu warnen, zu der das Buch, zumal in Folge der Verhältnisse, unter donen es entstand, verloiten könnte. Dieser Pflicht konnte sich der Roferent bei aller Werthschätzung der umfassenden and emsigen Thätigkeit des Verfassers nicht entziehon, dessen Bemühungen damit ebenso anerkannt seien, wie die Opferwilligkeit der Verlagsbuchhandlung, welche das Buch auf das reichste und beste ansstattete.

\title{
Flora von 0esterreich-Ungarn.
}

\author{
A. Referate. \\ I. $\mathrm{B}$ ö $\mathrm{h} \mathrm{me} \mathrm{n}^{2}$ ), \\ Referent L. Člakorskîy (Prag). ${ }^{3}$ ) \\ Quellen:
}

P. Ascherson und P. Maguus. Die weisso Heidelbeere etc. in Ber. d. dentsch. bot. Gosellsch. 1889, Bd. VII, Hft. 10 (1).

L. Celakovský. Uebor Petasites Kublikianus Tausch. Oesterr. bot. Zeitschr. $1890 \mathrm{Nr} .7$ und 8 (2).

E. Fiek. Resultate der Durchforschung der schlesischen Phanerogamentlora im Jahre 1889 (3).

R. Glaser. "Prag." Beiblätter zu „Ost und West" 1844 Nr. 117. Corresp. "aus Gitschin" (4).

Originalbeiträge (Verzeichnisse nnd Pflanzensendungen) von Fr. Bubák (5), V. v. Cypers (6), Jos. Jahn (7), W. Hess (8),

1) Als solche Einzelheiten sind beispielsweise gemeint: Die Angabe, dass die weiblichen Blüthenzapfen der gewöhnlichen Lärche "grünlich-weiss" seien; die Benennung einer sandliebenden Pflanze als "psammophylla" (statt psammophila etc. etc.

$\left.{ }^{2}\right)$ Das Referat behandelt den Zeitraum rom 1. Juni 1890 bis 1. Jänner 1891.

3) Beschreibungen der neuen Formen und überhaupt der für das Gebiet neuen Pflanzenformen werden in den demnächst erscheinenden „Resultaten der botanischen Durchforschung Böhmens für 1890" gegeben, deren auf das Wichtigste beschränkter Auszug das vorliegende Referat ist. 